\title{
Effect of irrigation regime on the production of volatiles that affect the aroma of the pear variety Triumph of Vienna (Pyrus communis L.)
}

\section{Efecto del régimen de riego en la producción de volátiles que incide en el aroma de la pera variedad Triunfo de Viena (Pyrus communis L.)}
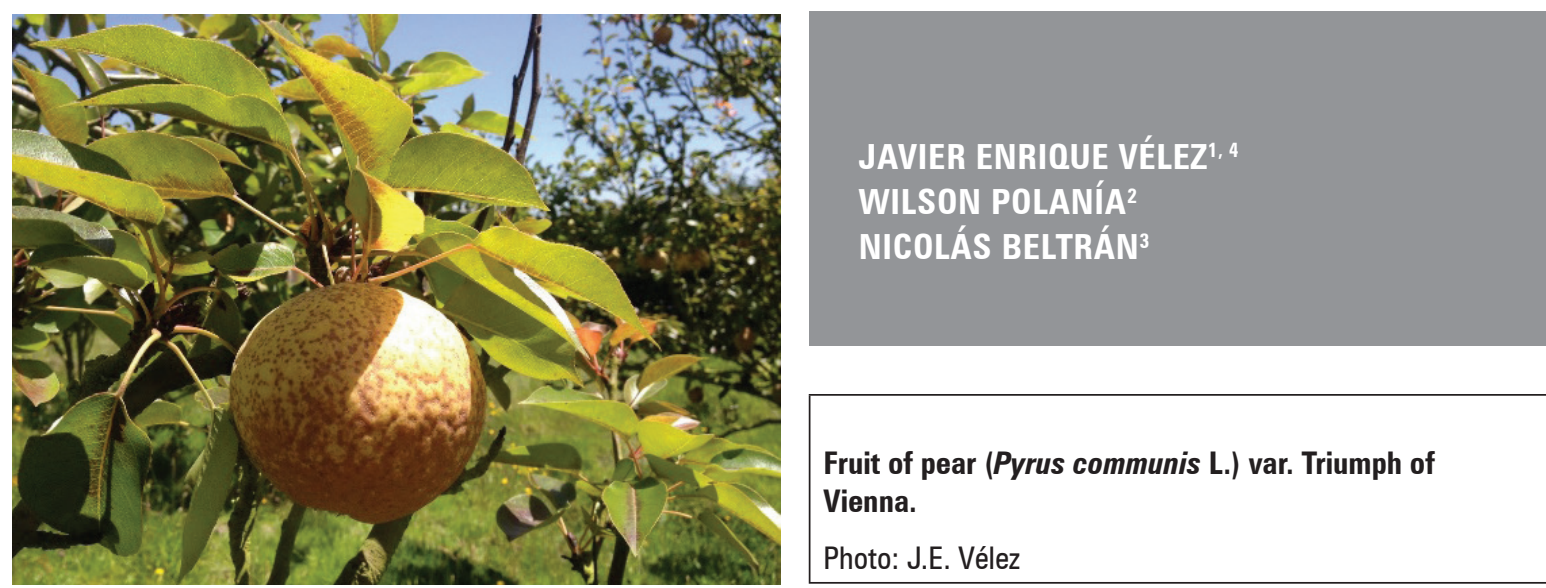

Fruit of pear (Pyrus communis L.) var. Triumph of Vienna.

Photo: J.E. Vélez

\section{ABSTRACT}

Water is a major component of plants that directly and indirectly affects physiological processes. One of the consequences of a hydric deficit in the pear fruit is modification of the aroma. No information exists on the effect of a water deficit on the sensory profile and volatile composition of this species. The objective was to determine the production of volatiles in the harvest and post-harvest of pear var. Triumph of Vienna (Pyrus communis $\mathrm{L}$.) with regulated deficit irrigation (RDI). The irrigation treatments consisted of the application of water regimes that were 100 (Control), 74 and $48 \%$ of the ETc during the rapid fruit growth period. The rest of the season plants-were irrigated at $100 \% \mathrm{ETc}$. In the deficit treatments, there were no significant reductions with respect to the control in the quality of the fruits, obtaining a water savings in 74 and $48 \%$ ETc of 26 and $40 \%$, respectively. The esters were the volatile compounds that contribute greatly to aroma, which increased steadily during the climacteric phase. Under the limited water conditions, watering with regulated, deficit doses obtained production that was similar to that of well-watered crops, provided that it was carried out in the phenological stage of low sensitivity and that the tolerance limits of stress were not exceeded.

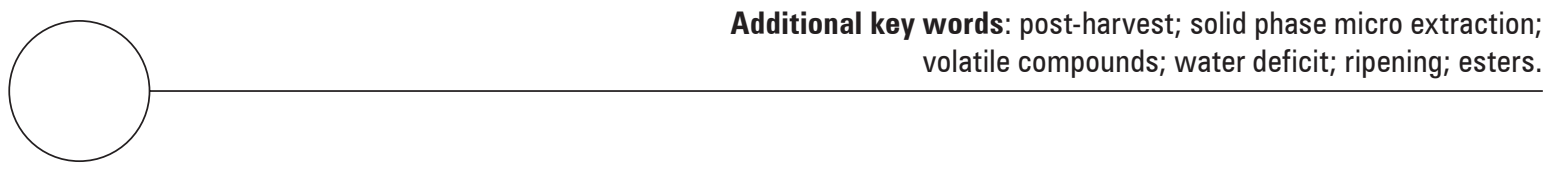

1 Universidad Nacional de Colombia, Faculty of Engineering, Department of Civil and Agricultural, Bogota (Colombia). ORCID Vélez, J.E.: 0000-0002-1361-8374

2 Universidad Nacional de Colombia, Faculty of Sciences, Department of Chemistry, Bogota (Colombia). ORCID Polanía, W.: 0000-0003-2355-9408

3 Columbia University, Columbia College, New York, EE UU, ORCID Beltrán, N.: 0000-0001-8217-3092

4 Corresponding author. jevelezs@unal.edu.co 


\section{RESUMEN}

El agua es el componente mayoritario de la planta que afecta directa e indirectamente los procesos fisiológicos. Uno de los efectos del déficit hídrico en el fruto de la pera es la modificación del aroma y no existe información sobre el efecto del déficit hídrico en el perfil sensorial y composición de volátiles de esta especie. El objetivo fue determinar la producción de volátiles en la cosecha y poscosecha del peral var. Triunfo de Viena (Pyrus communis L.) con riego deficitario regulado (RDR). Los tratamientos de riego consistieron en la aplicación de láminas de agua correspondiente al 100 (Control) 74 y 48\% de la ETc, durante el periodo de crecimiento rápido del fruto, el resto de la temporada se regaron al 100\% de la ETc. En los tratamientos deficitarios no hubo reducciones significativas respecto al control en la calidad de la fruta, obteniéndose un ahorro de agua en 74 y $48 \%$ ETc de 26 y $40 \%$, respectivamente. Los ésteres fueron los compuestos volátiles con mayor contribución al aroma que aumentaron de forma constante durante la fase del climaterio. En condiciones limitantes de agua, regar con dosis deficitarias controladas permite obtener producciones similares a las de un cultivo bien regado, siempre que se realice en el estado fenológico de baja sensibilidad y los límites tolerables de estrés no se superen.

Additional key words: poscosecha; micro extracción en fase sólida; compuestos volátiles; déficit de agua; maduración; ésteres.

Received for publication: 07-09-2019 Accepted for publication: 29-11-2019

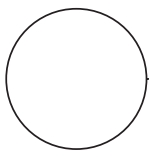

INTRODUCTION

The pear tree in Colombia in recent years has had an increase in yield, $15.8 \mathrm{t} \mathrm{ha}^{-1}$ in 2018 . It ranks second in terms of importance, after the peach (Agronet, 2019). This increase resulted from an increase in the consumption of fresh and processed fruits because of the recognition of nutritional and medicinal properties (Miranda et al., 2013).

Water consumption in agriculture represents about $87 \%$ of the global total, and demand is increasing. The depletion of water resources, the high costs of water and energy, the increase in demand, the decrease in international prices of fruit and the globalization of markets require improvements in the efficiency of crop production and irrigation (UNESCO, 2015).

Moderate water deficits during filling and maturation may be benefit internal changes in fruit quality, mainly by increasing the content of soluble solids and acids (Morandi et al., 2014; Galindo et al., 2017; Griñan et al., 2019). The pear is a climacteric fruit that produces greater amounts of ethylene and intense aroma during ripening that affect sensory quality and, consequently, consumer satisfaction, with a complex mixture of many volatile compounds that includes terpenes, alcohols, aldehydes, esters, acids, ketones and hydrocarbons, whose concentrations determine organoleptic attributes and consumer preference (Li et al., 2012).
This is the first study aimed at determining the effect on the production of volatiles in the harvest and post-harvest periods of the pear Triumph of Vienna cultivar (Pyrus communis L.) of regulated deficit irrigation (RDI) during the rapid growth stage of the fruit in 2014, taking into account the water status of the soil and the plants.

\section{MATERIAL AND METHODS}

This experiment was carried out in 2014 in Hacienda San Benito of Sesquile, Cundinamarca, Colombia. The plot had an area of 0.32 ha, with 172 pear trees of the Triumph of Vienna cultivar (Pyrus communis L.), planted in 1998 at $4 \times 4 \mathrm{~m}$. The soil has a loamy texture (IGAC, 2010). The average temperature was $12^{\circ} \mathrm{C}$. The total precipitation in the period from November 2013 to April 2014 was $465.4 \mathrm{~mm}$. The annual average evapotranspiration ETo, calculated with the Thornthwaite method, was $650 \mathrm{~mm}$. The average daily ETc, determined with the Penman-Monteith equation (Allen et al., 1998; Cleves et al., 2016) with crop coefficient $\mathrm{Kc}=0.8$, was $2.16 \mathrm{~mm} \mathrm{~d}^{-1}$, and the relative humidity (RH) was $78.2 \%$.

The drip irrigation system used six emitters per tree, $8 \mathrm{~L} \mathrm{~h}^{-1}$. The experiment design was randomized 
complete blocks, taking into account the slope of the land and the distribution of the trees in the plot, with three treatments and four repetitions per treatment (12 plots). The experiment plot was formed by 4 or 5 contiguous rows of three, four or five trees (with a total of 12, 15, 16 and 20 interior trees per plot).

The irrigation regime was determined by considering the crop evapotranspiration (ETc). During the cultivation cycle, all treatments were irrigated at $100 \%$ of the ETc, and, from January 1 to February 28, 2014, during the rapid growth phase, the following treatments were applied: control of $100 \%$ ETc irrigated all year and deficit treatments (74\%ETc and 48\%ETc) irrigated at 74 and $48 \%$ of the control, respectively. The volume of water applied to each treatment was regulated by varying the irrigation time, maintaining the same frequency, every $2 \mathrm{~d}$. The water was measured with $13 \mathrm{~mm}$ volumetric meters, Zenner ${ }^{\circledR}$, installed in each plot.

From a random sample of each repetition on May 9, 2014 to harvest, May 21 (12 days after harvesting DAH) and (24 DAH) June 2, two complete fruits were obtained to determine the volatiles using the SPME solid phase micro extraction methodology (Stashenko and Martínez, 2011; Li et al., 2012; CanoLamadrid et al., 2018) with a 1,160 mL storage vial.

The head space calculation for each of the tests was determined as the volume of the vial minus the volume of the fruit (Tab. 1). The standardization of the method was carried out by means of a time of exposure of the fiber to the volatiles for $30 \mathrm{~min}$ at an approximate ambient temperature between 22 and $26^{\circ} \mathrm{C}$. The fiber was Supelco brand, three phases (divinil benzene, carboxen and polydimethylsiloxane) with an equilibrium time of $1 \mathrm{~h}$. The internal standard used 2-octanol, $5.0 \mu \mathrm{L}$.

The fiber was conditioned with thermal desorption for $5 \mathrm{~min}$ in a gas chromatograph (GC), Agilent Technologies 7890A injection port coupled to a 5975C mass spectrometer (MS) (Santa Clara, Ca), at a temperature of $250^{\circ} \mathrm{C}$ for $30 \mathrm{~min}$. The operating conditions used an Agilent 19091S-433 high resolution gas chromatography column ( $30 \mathrm{~m} \times 250 \mathrm{~mm}$ x $0.25 \mu \mathrm{m}$ stationary phase film). Helium ( $\mathrm{He}$ ) gas was used as the carrier with a column flow of $1 \mathrm{~mL}$ $\mathrm{min}^{-1}$, with a running time of $51 \mathrm{~min}$ and at a 1:50 splitless mode ratio. The temperature of the injector and the interface was $250^{\circ} \mathrm{C}$. For the acquisition of the mass spectrometer data, Chem Station software was used, and the mass spectra were compared with those stored in the NIST library to identify the compounds.

With the SAS/STAT program (SAS Institute, 2010), the statistical analysis was carried out using analysis of variance and Tukey test, $P \leq 0.05$, to compare the treatments and the control with the ' $\mathrm{glm}$ ' procedure.

Table 1. Weight and volume of the fruit and head space in the vial for each treatment and repetition at harvest, 12 and 24 DAH in 2014.

\begin{tabular}{|l|c|c|c|c|c|c|c|c|c|}
\hline \multirow{2}{*}{ Treatment } & \multicolumn{3}{|c|}{ May 9 (harvest) } & \multicolumn{3}{c|}{ May 21 (12 DAH) } & \multicolumn{3}{c|}{ June 2 (24 DAH) } \\
\cline { 2 - 11 } & $\begin{array}{c}\text { Weight } \\
\text { (g) }\end{array}$ & $\begin{array}{c}\text { Volume } \\
\text { (cc) }\end{array}$ & $\begin{array}{c}\text { Head space } \\
\text { (cc) }\end{array}$ & $\begin{array}{c}\text { Weight } \\
\text { (g) }\end{array}$ & $\begin{array}{c}\text { Volume } \\
\text { (cc) }\end{array}$ & $\begin{array}{c}\text { Head space } \\
\text { (cc) }\end{array}$ & $\begin{array}{c}\text { Weight } \\
\text { (g) }\end{array}$ & $\begin{array}{c}\text { Volume } \\
\text { (cc) }\end{array}$ & $\begin{array}{c}\text { Head space } \\
\text { (cc) }\end{array}$ \\
\hline $100 \%$ ETCR1 & 280.26 & 276.02 & 883.98 & 173.28 & 179.19 & 980.81 & 285.98 & 281.20 & 878.80 \\
\hline $100 \%$ ETCR2 & 229.00 & 229.62 & 930.38 & 174.49 & 180.28 & 979.72 & 244.14 & 243.32 & 916.68 \\
\hline $100 \%$ ETCR3 & 212.46 & 214.65 & 945.35 & 178.31 & 183.74 & 976.26 & 277.68 & 273.68 & 886.32 \\
\hline $100 \%$ ETCR4 & 274.36 & 270.68 & 889.32 & 213.03 & 215.17 & 944.83 & 299.68 & 293.60 & 866.40 \\
\hline $74 \%$ ETCR1 & 267.64 & 265.27 & 894.73 & 220.09 & 221.14 & 938.86 & 327.72 & 321.02 & 838.98 \\
\hline $74 \%$ ETCR2 & 207.37 & 209.34 & 950.66 & 161.58 & 166.85 & 993.15 & 271.79 & 269.12 & 890.88 \\
\hline $74 \%$ ETCR3 & 212.32 & 213.93 & 946.07 & 197.70 & 200.37 & 959.63 & 290.64 & 286.61 & 873.39 \\
\hline $74 \%$ ETCR4 & 226.00 & 226.63 & 933.37 & 181.54 & 185.37 & 974.63 & 249.60 & 248.53 & 911.47 \\
\hline $48 \%$ ETCR1 & 253.16 & 248.43 & 911.57 & 213.40 & 214.10 & 945.90 & 243.91 & 240.44 & 919.56 \\
\hline $48 \%$ ETCR2 & 202.97 & 205.09 & 954.91 & 226.77 & 225.64 & 934.36 & 294.67 & 284.27 & 875.73 \\
\hline $48 \%$ ETCR3 & 179.78 & 185.07 & 974.93 & 138.14 & 149.12 & 1010.88 & 288.20 & 278.68 & 881.32 \\
\hline $48 \%$ ETCR4 & 181.02 & 186.14 & 973.86 & 136.32 & 147.55 & 1012.45 & 255.44 & 250.40 & 909.60 \\
\hline
\end{tabular}




\section{RESULTS AND DISCUSSION}

The chromatogram standardization method of the volatile fraction (Fig. 1) corresponded to one of the repetitions of the $100 \%$ ETc treatment at harvest, where the more abundant peaks were identified in the retention times (tr), which were: (1) methyl acetate, $\operatorname{tr}=2.2 \mathrm{~min}(5.20,2.26$ and $4.19 \%)$; (2) butyl acetate, $\operatorname{tr}=5.7 \mathrm{~min}(12.29,12.52$ and $14.46 \%) ;(3)$ ethyl hexanoate, $\operatorname{tr}=8.8 \mathrm{~min}(3.95,4.49$ and $7.13 \%)$ and (4) octanol, tr $=16.38 \mathrm{~min}(18.18,24.43$ and $18.22 \%)$. The determinations were made at harvest and 12 and $24 \mathrm{DAH}$ for each treatment and repetition, whose mass spectra were compared with those stored in the NIST library.

Twenty-three (23) volatile compounds were found, including 2-octanol, used as an internal standard, equal to the number of volatiles identified by $\mathrm{Li}$ et al. (2014) in the pear 'Pingxiangli' in different stages of maturation. The sensory descriptors correspond to SAFC (2011), which have also been reported in pear and other fruit trees by Altisent et al. (2011), Verzera et al. (2011) and Griñan et al. (2019).

The total concentration in the percentage of area of the volatile was $53.72,56.69$ and $60.92 \%$ at the time of harvest for $100 \% \mathrm{ETc}, 74 \% \mathrm{ETc}$ and $48 \% \mathrm{ETc}$, respectively, with no significant statistical differences although the deficient treatment 48\%ETC was higher (Tab. 2).

The 12 DAH concentration increased to $87.26 ; 89.70$ and $86.08 \%$, respectively, with no difference between treatments. While, the $24 \mathrm{DAH}$ concentration decreased, with a difference between treatments 100 and $74 \% E T c$, with a total concentration of 85.62 ; 66.86 and $79.56 \%$ for $100 \% E T c, 74 \% E T c$ and $48 \% E T c$, respectively (Tab. 2).

The dominant compounds in percentage of area for 12 DAH were ethyl acetate, propyl acetate, butyl acetate, hexyl acetate, octanol and $\alpha$-farnese, similar to the compounds found by Zlatic et al. (2016) in the 'Bartlett' pear and by Bhavadharani et al. (2019) in the pear fruit (Pyrus communis).

According to the percentages of areas corresponding to the chromatograms and the weight of the fruits, the content of the volatile compounds, which contribute to the aroma of the pear cultivar Triumph of Vienna, was calculated, with a total concentration in $100 \% \mathrm{ETc}, 74 \% \mathrm{ETc}$ and $48 \% \mathrm{ETc}$, without differences for the harvest at $38.14 ; 23.65$ and $43.38 \mathrm{mg} \mathrm{kg}^{-1} \mathrm{~L}^{-1}$;

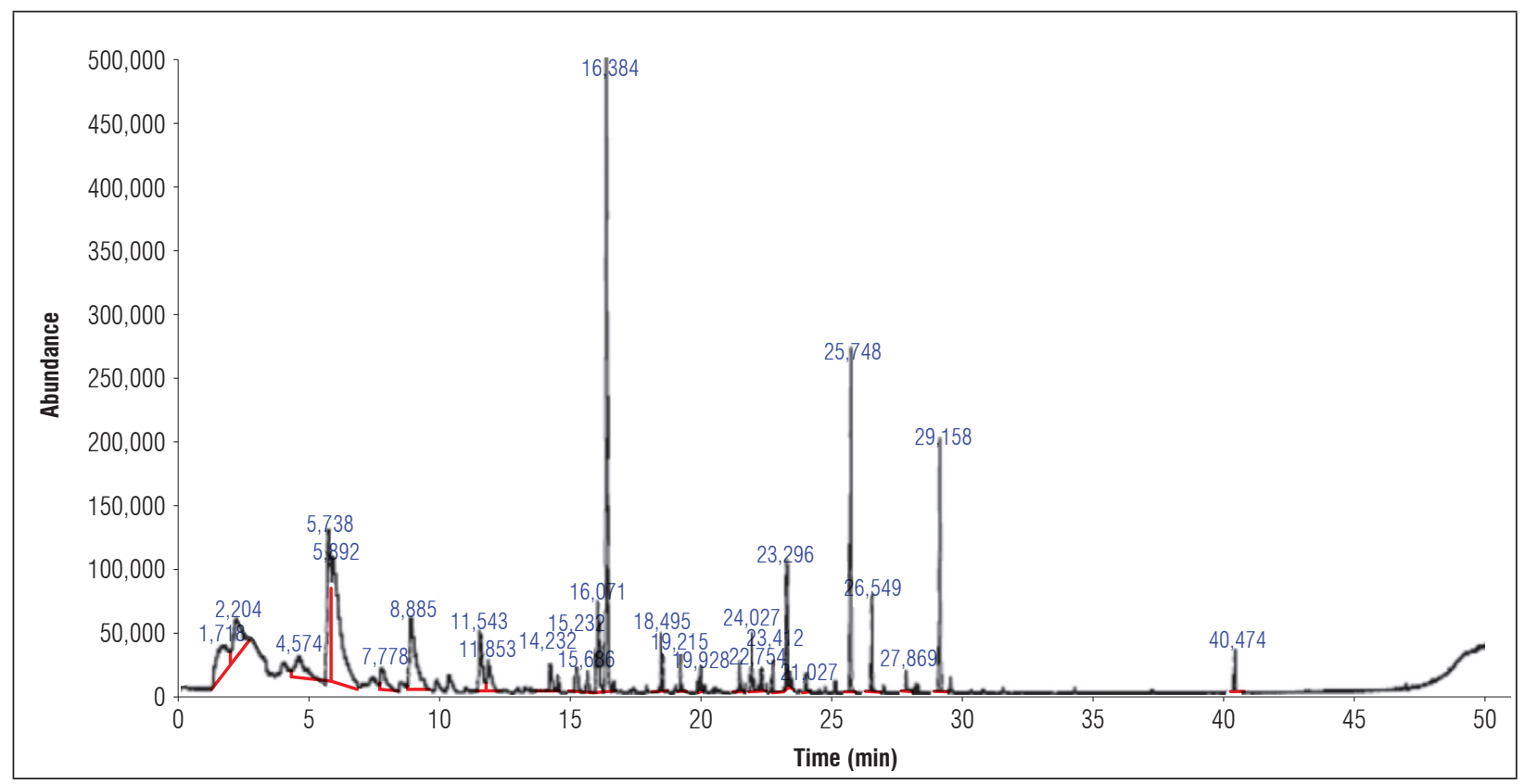

Figure 1. Chromatogram of the qualitative analysis of the volatile compounds constituting the characteristic aroma of the pear variety Triumph of Vienna in $100 \%$ ETc treatment at harvest in 2014, with four of the peaks identified: (1) methyl acetate, $\operatorname{tr}=2.2 \mathrm{~min}$; (2) butyl acetate, $\operatorname{tr}=5.7 \mathrm{~min}$; (3) ethyl hexanoate, $\operatorname{tr}=8.8 \mathrm{~min}$ and (4) octanol, $\operatorname{tr}=16.38 \mathrm{~min}$. 
$12 \mathrm{DAH}$ increased to $237.49,219.96$ and $296.60, \mathrm{mg}$ $\mathrm{kg}^{-1} \mathrm{~L}^{-1}$; and 24 DAH decreased to 227.31, 151.67 and $153.41 \mathrm{mg} \mathrm{kg}^{-1} \mathrm{~L}^{-1}$ (Tab. 3). The production of volatile compounds increased during maturation and slowed down at $24 \mathrm{DAH}$, possibly because of a restricted supply of substrates for esterification, similar to that found by Bangerth et al. (2012) in apples. This behavior is typical of climacteric fruits, which produce a greater quantity of compounds that are characteristic of intense aromas during ripening, coinciding with a high production of ethylene and high respiration, which produce several physiological changes.

The content of volatiles in $\mathrm{mg} \mathrm{kg}^{-1} \mathrm{~L}^{-1}$ in general did not differ between the treatments, except: at harvest between $74 \% \mathrm{ETc}$ and $48 \% \mathrm{ETc}$ for methyl hexanoate; 12 DAH between $74 \%$ ETc and $48 \%$ ETc for ethanal; $100 \% \mathrm{ETc}$ and $48 \% \mathrm{ETc}$ for butyl acetate and $100 \% \mathrm{ETC}$ with (74\%ETc and 48\%ETc) for 2-methylbutyl acetate (Tab. 3).

The total volatile content increased in the sampling point, with statistical differences in the $100 \% \mathrm{ETc}$ treatment, from 38.14 to $237.49 \mathrm{mg} \mathrm{kg}^{-1} \mathrm{~L}^{-1}$ between the harvest and $12 \mathrm{DAH}$, respectively, and without differences between 12 and $24 \mathrm{DAH}$; the 74\%ETc treatment had differences, with values of 219.96, 151.67 and $23.65 \mathrm{mg} \mathrm{kg}^{-1} \mathrm{~L}^{-1}, 12,24 \mathrm{DAH}$ and harvest, respectively; in $48 \% \mathrm{ETc}, 12 \mathrm{DHA}$ showed differences from the other sampling points (Tab. 4).

Table 2. Percentage of area of the volatile compounds in the pear Triumph of Vienna cultivar in 2014.

\begin{tabular}{|c|c|c|c|c|c|c|c|c|c|c|}
\hline \multirow{2}{*}{$\begin{array}{l}\text { Volatile } \\
\text { (\% area) }\end{array}$} & \multirow{2}{*}{$\begin{array}{l}\text { Time } \\
\text { (min) }\end{array}$} & \multicolumn{3}{|c|}{ May 9 (harvest) } & \multicolumn{3}{|c|}{ May 21 (12 DAH) } & \multicolumn{3}{|c|}{ June 2 (24 DAH) } \\
\hline & & $100 \% \mathrm{ETc}$ & $74 \% \mathrm{ETc}$ & $48 \% \mathrm{ETc}$ & $100 \% \mathrm{ETc}$ & $74 \% \mathrm{ETc}$ & $48 \% \mathrm{ETc}$ & $100 \% \mathrm{ETc}$ & $74 \%$ ETc & $48 \% \mathrm{ETc}$ \\
\hline Pentanal & 1.69 & $2.70 \mathrm{a}$ & $0.00 \mathrm{a}$ & $2.15 a b$ & $0.00 \mathrm{a}$ & $0.00 \mathrm{a}$ & $0.00 \mathrm{a}$ & $0.00 \mathrm{a}$ & $0.00 \mathrm{a}$ & $0.00 \mathrm{a}$ \\
\hline Ethanal & 1.70 & $1.70 \mathrm{a}$ & $2.34 \mathrm{a}$ & $4.93 \mathrm{bc}$ & $0.53 \mathrm{a}$ & $1.68 a b$ & $0.00 \mathrm{a}$ & $1.10 \mathrm{a}$ & $1.10 \mathrm{a}$ & $1.37 a b$ \\
\hline methyl acetate & 2.20 & $5.20 \mathrm{a}$ & $2.26 \mathrm{a}$ & $4.19 \mathrm{abc}$ & $1.75 \mathrm{ab}$ & $2.35 \mathrm{abc}$ & $2.15 a b$ & $2.03 \mathrm{a}$ & $1.14 \mathrm{a}$ & $1.46 a b$ \\
\hline Ethyl acetate & 2.73 & $0.00 \mathrm{a}$ & $0.00 \mathrm{a}$ & $0.00 \mathrm{a}$ & $24.25 \mathrm{e}$ & $15.30 \mathrm{e}$ & $15.32 \mathrm{~d}$ & 13.08 bc & $5.70 \mathrm{ab}$ & $9.15 b$ \\
\hline Ethyl Propanoate & 3.53 & $0.00 \mathrm{a}$ & $0.00 \mathrm{a}$ & $0.00 \mathrm{a}$ & $0.00 \mathrm{a}$ & $0.73 a$ & $0.00 \mathrm{a}$ & $0.48 \mathrm{a}$ & $0.58 \mathrm{a}$ & $0.00 \mathrm{a}$ \\
\hline Propyl acetate & 3.80 & $0.00 \mathrm{a}$ & $0.00 \mathrm{a}$ & $0.00 \mathrm{a}$ & $8.45 \mathrm{c}$ & $6.32 \mathrm{~cd}$ & $6.28 \mathrm{c}$ & $1.34 \mathrm{a}$ & $1.03 \mathrm{a}$ & $1.24 \mathrm{a}$ \\
\hline Ethyl butanoate & 4.97 & $0.00 \mathrm{a}$ & $0.00 \mathrm{a}$ & $0.00 \mathrm{a}$ & $4.12 \mathrm{abc}$ & $3.37 \mathrm{abc}$ & $3.08 a b$ & $3.39 \mathrm{a}$ & $1.93 \mathrm{a}$ & $2.77 a b$ \\
\hline Butyl acetate & 5.70 & $12.29 b$ & $12.52 b$ & $14.46 \mathrm{~d}$ & $17.11 \mathrm{~d}$ & $24.04 \mathrm{f}$ & $21.86 \mathrm{e}$ & $24.07 d$ & $23.60 \mathrm{c}$ & $31.05 c$ \\
\hline 2-methylbutyl acetate & 7.01 & $0.00 \mathrm{a}$ & $0.00 \mathrm{a}$ & $0.00 \mathrm{a}$ & $1.17 \mathrm{a}$ & $2.44 a b c$ & $2.02 a b$ & $0.63 a$ & $0.73 a$ & $0.59 a$ \\
\hline 3-methylbutyl acetate & 7.02 & $0.00 \mathrm{a}$ & $0.00 \mathrm{a}$ & $0.00 \mathrm{a}$ & $0.00 \mathrm{a}$ & $0.23 a$ & $0.43 a$ & $0.70 \mathrm{a}$ & $0.64 \mathrm{a}$ & $0.00 \mathrm{a}$ \\
\hline Pentyl acetate & 8.40 & $0.00 \mathrm{a}$ & $0.00 \mathrm{a}$ & $0.00 \mathrm{a}$ & $0.44 \mathrm{a}$ & $0.46 \mathrm{a}$ & $0.16 \mathrm{a}$ & $0.42 \mathrm{a}$ & $0.41 \mathrm{a}$ & $1.20 a b$ \\
\hline Heptyl acetate & 8.49 & $0.00 \mathrm{a}$ & $0.00 \mathrm{a}$ & $0.00 \mathrm{a}$ & $0.19 a$ & $0.24 \mathrm{a}$ & $0.16 \mathrm{a}$ & $0.00 \mathrm{a}$ & $0.43 \mathrm{a}$ & $0.00 \mathrm{a}$ \\
\hline methyl hexanoate & 8.80 & $3.95 \mathrm{a}$ & $4.49 a$ & $7.13 \mathrm{c}$ & $1.46 \mathrm{a}$ & $1.77 a b$ & $1.72 a b$ & $2.95 \mathrm{a}$ & $2.52 \mathrm{a}$ & $2.36 a b$ \\
\hline $\begin{array}{l}\text { Ethyl hexanoate-ethyl } \\
\text { ester }\end{array}$ & 10.30 & $0.00 \mathrm{a}$ & $0.41 \mathrm{a}$ & $0.00 \mathrm{a}$ & $4.26 a b c$ & $4.07 \mathrm{abcd}$ & $3.72 b$ & $6.49 a b$ & $2.80 \mathrm{a}$ & $6.50 a b$ \\
\hline Hexyl acetate & 11.50 & $0.94 \mathrm{a}$ & $1.20 \mathrm{a}$ & $0.81 \mathrm{ab}$ & $7.05 \mathrm{bc}$ & $7.33 \mathrm{~d}$ & $8.02 \mathrm{c}$ & $6.38 \mathrm{ab}$ & $5.71 a b$ & $5.99 a b$ \\
\hline Octanone & 11.80 & $2.93 \mathrm{a}$ & $3.52 \mathrm{a}$ & $2.69 a b$ & $0.21 \mathrm{a}$ & $0.21 \mathrm{a}$ & $0.00 \mathrm{a}$ & $0.31 \mathrm{a}$ & $0.34 \mathrm{a}$ & $0.55 a$ \\
\hline Hexanol & 14.20 & $0.43 a$ & $0.54 \mathrm{a}$ & $0.27 \mathrm{a}$ & $0.50 \mathrm{a}$ & $0.69 a$ & $0.44 \mathrm{a}$ & $0.68 \mathrm{a}$ & $0.72 \mathrm{a}$ & $0.96 a b$ \\
\hline hexyl butanoate & 16.07 & $2.65 \mathrm{a}$ & $1.90 \mathrm{a}$ & $2.36 a b$ & $0.35 \mathrm{a}$ & $0.38 \mathrm{a}$ & $0.94 a b$ & $2.18 \mathrm{a}$ & 1.58 & $1.57 a b$ \\
\hline Octanol & 16.38 & $18.18 \mathrm{c}$ & $24.43 \mathrm{c}$ & $18.22 \mathrm{~d}$ & $4.37 \mathrm{abc}$ & $4.69 \mathrm{bcd}$ & $3.80 \mathrm{~b}$ & $3.44 \mathrm{a}$ & $3.92 \mathrm{a}$ & $5.81 \mathrm{ab}$ \\
\hline ethyl octanoate & 16.66 & $0.00 \mathrm{a}$ & $0.00 \mathrm{a}$ & $0.00 \mathrm{a}$ & $0.49 \mathrm{a}$ & $0.46 \mathrm{a}$ & $0.51 \mathrm{a}$ & $0.22 \mathrm{a}$ & $0.23 \mathrm{a}$ & $0.41 \mathrm{a}$ \\
\hline ethyl hexanoate & 18.40 & $2.14 \mathrm{a}$ & $2.70 \mathrm{a}$ & $1.93 a b$ & $0.42 \mathrm{a}$ & $0.40 \mathrm{a}$ & 0.37 a & $0.30 \mathrm{a}$ & $0.43 \mathrm{a}$ & $0.43 \mathrm{a}$ \\
\hline ethyl decanoate & 22.75 & $0.00 \mathrm{a}$ & $0.00 \mathrm{a}$ & $0.15 a$ & $0.58 \mathrm{a}$ & 0.37 a & $0.62 \mathrm{a}$ & $0.21 \mathrm{a}$ & $0.16 \mathrm{a}$ & $0.10 \mathrm{a}$ \\
\hline$\alpha$ farnese & 25.70 & $0.62 \mathrm{a}$ & $0.39 a$ & $1.64 \mathrm{ab}$ & $9.60 \mathrm{c}$ & $12.19 \mathrm{e}$ & $14.50 \mathrm{~d}$ & $15.22 \mathrm{c}$ & $11.22 \mathrm{~b}$ & $6.09 a b$ \\
\hline Total & & $53.72 \mathrm{a}$ & 56.69 a & $60.92 \mathrm{a}$ & $87.26 \mathrm{a}$ & $89.70 \mathrm{a}$ & 86.08 a & 85.62 b & 66.86 a & $79.56 \mathrm{ab}$ \\
\hline
\end{tabular}

Means with different letters in the same row indicate significant statistical differences within each day, between treatment, according to the Tukey test $(P \leq 0.05$; $n=4)$. 
The total content of the $48 \% \mathrm{ETc}$ deficit treatment at harvest and during the initial storage process was greater, with significant differences (43.38 to 296.60 $\left.\mathrm{mg} \mathrm{kg}^{-1} \mathrm{~L}^{-1}\right)$. Table 4 shows the differences in the volatile content.

$100 \%$ ETc showed different statistics between harvest and 12 DAH for: propyl acetate; butyl acetate; 2-methylbutyl acetate; hexyl acetate; octanone; hexanol; ethyl octanoate and ethyl decanoate; between harvest and 24 DAH for: butyl acetate; 2-methylbutyl acetate; methyl hexanoate; hexyl acetate; octanone; hexanol; ethyl hexanoate and $\alpha$-farnese; and between 12 and 24 DAH for: propyl acetate and methyl hexanoate.
$74 \% E T c$ had differences between harvest and 12 DAH for: methyl acetate; ethyl acetate; propyl acetate; ethyl butanoate; butyl acetate; 2-methylbutyl acetate; methyl hexanoate; hexyl acetate; hexanol; octanol; ethyl octanoate; ethyl decanoate and $\alpha$ farnese; between harvest and 24 DAH for: butyl acetate; methyl hexanoate; hexyl acetate; hexanol and $\alpha$ farnese; and between 12 and 24 DAH for: methyl acetate; ethyl acetate; propyl acetate; 2-methylbutyl acetate; octanol and ethyl decanoate.

48\%ETc had differences between harvest and 12 DAH for: ethyl acetate; propyl acetate; ethyl butanoate; butyl acetate; 2-methylbutyl acetate; hexyl acetate; octanone; ethyl octanoate and $\alpha$-farnese;

Table 3. Content of volatiles of the treatments at harvest, 12 DAH and 24 DAH of the pear cultivar Triumph of Vienna.

\begin{tabular}{|c|c|c|c|c|c|c|c|c|c|c|}
\hline \multirow{2}{*}{$\begin{array}{c}\text { Volatile } \\
\left(\mathrm{mg} \mathrm{kg}^{-1} \mathrm{~L}^{-1}\right)\end{array}$} & \multirow{2}{*}{$\begin{array}{l}\text { Time } \\
\text { (min) }\end{array}$} & \multicolumn{3}{|c|}{ May 9 (harvest) } & \multicolumn{3}{|c|}{ May 21 (12 DAH) } & \multicolumn{3}{|c|}{ June 2 (24 DAH) } \\
\hline & & $100 \%$ ETc & $74 \%$ ETc & $48 \% \mathrm{ETC}$ & $100 \%$ ETc & $74 \%$ ETc & $48 \% E T c$ & $100 \%$ ETc & $74 \% \mathrm{ETc}$ & 48\%ETc \\
\hline Pentanal & 1.69 & $1.84 \mathrm{a}$ & $0.00 \mathrm{a}$ & $1.56 \mathrm{a}$ & $0.00 \mathrm{a}$ & $0.00 \mathrm{a}$ & $0.00 \mathrm{a}$ & $0.00 \mathrm{a}$ & $0.00 \mathrm{a}$ & $0.00 \mathrm{a}$ \\
\hline Ethanal & .70 & $1.54 \mathrm{a}$ & $1.27 \mathrm{a}$ & $2.84 \mathrm{a}$ & $1.07 \mathrm{ab}$ & $3.99 \mathrm{~b}$ & $0.00 \mathrm{a}$ & $2.83 \mathrm{a}$ & $2.27 \mathrm{a}$ & $2.07 \mathrm{a}$ \\
\hline Methyl acetate & 2.20 & $4.28 \mathrm{a}$ & $0.89 a$ & $3.37 \mathrm{a}$ & $4.56 \mathrm{a}$ & $5.67 \mathrm{a}$ & $7.09 \mathrm{a}$ & $4.83 \mathrm{a}$ & $2.51 \mathrm{a}$ & $2.80 \mathrm{a}$ \\
\hline Ethyl acetate & 2.73 & $0.00 \mathrm{a}$ & $0.00 \mathrm{a}$ & $0.00 \mathrm{a}$ & $69.00 \mathrm{a}$ & $38.80 \mathrm{a}$ & $54.73 \mathrm{a}$ & $41.62 \mathrm{a}$ & $12.80 \mathrm{a}$ & $24.88 \mathrm{a}$ \\
\hline Ethyl propanoate & 3.53 & $0.00 \mathrm{a}$ & $0.00 \mathrm{a}$ & $0.00 \mathrm{a}$ & $0.00 \mathrm{a}$ & $1.87 \mathrm{a}$ & $0.00 \mathrm{a}$ & $1.56 \mathrm{a}$ & $1.36 \mathrm{a}$ & $0.00 \mathrm{a}$ \\
\hline Propyl acetate & 3.80 & $0.00 \mathrm{a}$ & $0.00 \mathrm{a}$ & $0.00 \mathrm{a}$ & $23.47 \mathrm{a}$ & $15.66 \mathrm{a}$ & $21.46 \mathrm{a}$ & $4.34 \mathrm{a}$ & $2.04 \mathrm{a}$ & $3.29 \mathrm{a}$ \\
\hline Ethyl butanoate & 4.97 & $0.00 \mathrm{a}$ & $0.00 \mathrm{a}$ & $0.00 \mathrm{a}$ & $11.38 \mathrm{a}$ & $8.70 \mathrm{a}$ & $11.59 \mathrm{a}$ & $10.83 \mathrm{a}$ & $4.42 \mathrm{a}$ & $7.51 \mathrm{a}$ \\
\hline Butyl acetate & 5.70 & $9.62 \mathrm{a}$ & $5.34 \mathrm{a}$ & $10.50 \mathrm{a}$ & $45.66 \mathrm{a}$ & $57.31 \mathrm{ab}$ & $70.31 \mathrm{~b}$ & $58.85 \mathrm{a}$ & $57.21 \mathrm{a}$ & $48.59 \mathrm{a}$ \\
\hline 2-methylbutyl acetate & 7.01 & $0.00 \mathrm{a}$ & $0.00 \mathrm{a}$ & $0.00 \mathrm{a}$ & $2.92 \mathrm{a}$ & $5.94 b$ & $7.07 b$ & $1.53 \mathrm{a}$ & $1.43 \mathrm{a}$ & $1.10 \mathrm{a}$ \\
\hline 3-methylbutyl acetate & 7.02 & $0.00 \mathrm{a}$ & $0.00 \mathrm{a}$ & $0.00 \mathrm{a}$ & $0.00 \mathrm{a}$ & $0.70 \mathrm{a}$ & $1.10 \mathrm{a}$ & $1.70 \mathrm{a}$ & $1.26 \mathrm{a}$ & $0.00 \mathrm{a}$ \\
\hline Pentyl acetate & 8.40 & $0.00 \mathrm{a}$ & $0.00 \mathrm{a}$ & $0.00 \mathrm{a}$ & $1.08 \mathrm{a}$ & $1.07 \mathrm{a}$ & $0.67 \mathrm{a}$ & $1.18 \mathrm{a}$ & $0.80 \mathrm{a}$ & $2.06 \mathrm{a}$ \\
\hline Heptyl acetate & 8.49 & $0.00 \mathrm{a}$ & $0.00 \mathrm{a}$ & $0.00 \mathrm{a}$ & $0.50 \mathrm{a}$ & $0.49 a$ & $0.73 a$ & $0.00 \mathrm{a}$ & $1.24 \mathrm{a}$ & $0.00 \mathrm{a}$ \\
\hline Methyl hexanoate & 8.80 & $2.64 a b$ & $1.93 \mathrm{a}$ & $5.12 b$ & $3.92 \mathrm{a}$ & $4.35 \mathrm{a}$ & $5.90 \mathrm{a}$ & $7.54 \mathrm{a}$ & $5.63 a$ & $4.32 \mathrm{a}$ \\
\hline $\begin{array}{l}\text { Ethyl hexanoate-ethyl } \\
\text { ester }\end{array}$ & 10.30 & $0.00 \mathrm{a}$ & $0.22 \mathrm{a}$ & $0.00 \mathrm{a}$ & $11.66 \mathrm{a}$ & $10.61 \mathrm{a}$ & $13.82 \mathrm{a}$ & 20.61 a & $6.52 \mathrm{a}$ & $17.66 \mathrm{a}$ \\
\hline Hexyl acetate & 11.50 & $0.85 a$ & $0.56 \mathrm{a}$ & $0.60 \mathrm{a}$ & $19.20 \mathrm{a}$ & $18.51 \mathrm{a}$ & $27.48 \mathrm{a}$ & $17.68 \mathrm{a}$ & $13.26 \mathrm{a}$ & $13.44 \mathrm{a}$ \\
\hline Octanone & 11.80 & $1.83 \mathrm{a}$ & $1.41 \mathrm{a}$ & $1.90 \mathrm{a}$ & $0.42 \mathrm{a}$ & $0.46 \mathrm{a}$ & $0.00 \mathrm{a}$ & $0.61 \mathrm{a}$ & $0.59 a$ & $0.56 \mathrm{a}$ \\
\hline Hexanol & 14.20 & $0.39 a$ & $0.25 \mathrm{a}$ & $0.20 \mathrm{a}$ & $1.32 \mathrm{a}$ & $1.69 \mathrm{a}$ & $1.44 \mathrm{a}$ & $1.70 \mathrm{a}$ & $1.59 \mathrm{a}$ & $1.57 \mathrm{a}$ \\
\hline Hexyl butanoate & 16.07 & $2.03 \mathrm{a}$ & $0.80 \mathrm{a}$ & $1.72 \mathrm{a}$ & $0.97 \mathrm{a}$ & $0.94 \mathrm{a}$ & $3.35 \mathrm{a}$ & $4.57 \mathrm{a}$ & $2.77 \mathrm{a}$ & $2.01 \mathrm{a}$ \\
\hline Octanol & 16.38 & $11.24 \mathrm{a}$ & $9.73 \mathrm{a}$ & $12.93 \mathrm{a}$ & $11.51 \mathrm{a}$ & $11.28 \mathrm{a}$ & $12.35 \mathrm{a}$ & $8.39 \mathrm{a}$ & $8.26 \mathrm{a}$ & $8.50 \mathrm{a}$ \\
\hline Ethyl octanoate & 16.66 & $0.00 \mathrm{a}$ & $0.00 \mathrm{a}$ & $0.00 \mathrm{a}$ & $1.29 \mathrm{a}$ & $1.21 \mathrm{a}$ & $1.92 \mathrm{a}$ & $0.67 \mathrm{a}$ & $0.52 \mathrm{a}$ & $0.95 \mathrm{a}$ \\
\hline Ethyl hexanoate & 18.40 & $1.33 \mathrm{a}$ & $1.08 \mathrm{a}$ & $1.33 \mathrm{a}$ & $1.09 a$ & $0.96 \mathrm{a}$ & $1.21 \mathrm{a}$ & $0.70 \mathrm{a}$ & $0.90 \mathrm{a}$ & $0.67 \mathrm{a}$ \\
\hline Ethyl decanoate & 22.75 & $0.00 \mathrm{a}$ & $0.00 \mathrm{a}$ & $0.11 \mathrm{a}$ & $1.40 \mathrm{a}$ & $0.94 \mathrm{a}$ & $2.30 \mathrm{a}$ & $0.65 \mathrm{a}$ & $0.36 \mathrm{a}$ & $0.27 \mathrm{a}$ \\
\hline$\alpha$ farnese & 25.70 & $0.56 \mathrm{a}$ & $0.16 \mathrm{a}$ & $1.21 \mathrm{a}$ & $25.07 \mathrm{a}$ & $28.81 \mathrm{a}$ & $52.08 \mathrm{a}$ & $34.92 \mathrm{a}$ & $23.90 \mathrm{a}$ & $11.15 \mathrm{a}$ \\
\hline Total & & $38.14 \mathrm{a}$ & $23.65 \mathrm{a}$ & 43.38 a & 237.49 a & $219.96 \mathrm{a}$ & 296.60 a & $227.31 \mathrm{a}$ & $151.67 \mathrm{a}$ & $153.41 \mathrm{a}$ \\
\hline
\end{tabular}

Means with different letters in the same row indicate significant statistical differences within each sampling point, between treatment, according to the Tukey test $(P \leq 0.05 ; n=4)$. 
Table 4. Content of volatiles corresponding sampling point in $100 \% E T c, 74 \% E T c$ and $48 \% E T c$ of the pear cultivar Triumph of Vienna.

\begin{tabular}{|c|c|c|c|c|c|c|c|c|c|c|}
\hline \multirow{2}{*}{$\begin{array}{c}\text { Volatile } \\
\left(\mathrm{mg} \mathrm{kg}^{-1} \mathrm{~L}^{-1}\right)\end{array}$} & \multirow{2}{*}{$\begin{array}{l}\text { Time } \\
\text { (min) }\end{array}$} & \multicolumn{3}{|c|}{$100 \%$ ETc } & \multicolumn{3}{|c|}{ 74\%ETc } & \multicolumn{3}{|c|}{$48 \% \mathrm{ETc}$} \\
\hline & & $\begin{array}{c}\text { May } 9 \\
\text { (harvest) }\end{array}$ & $\begin{array}{c}\text { May } 21 \\
\text { (12 DAH) }\end{array}$ & $\begin{array}{c}\text { June } 2 \\
\text { (24 DAH) }\end{array}$ & $\begin{array}{c}\text { May } 9 \\
\text { (harvest) }\end{array}$ & $\begin{array}{c}\text { May } 21 \\
\text { (12 DAH) }\end{array}$ & $\begin{array}{c}\text { June } 2 \\
\text { (24 DAH) }\end{array}$ & $\begin{array}{c}\text { May } 9 \\
\text { (harvest) }\end{array}$ & $\begin{array}{c}\text { May } 21 \\
\text { (12 DAH) }\end{array}$ & $\begin{array}{c}\text { June } 2 \\
\text { (24 DAH) }\end{array}$ \\
\hline Pentanal & 1.69 & $1.84 \mathrm{a}$ & $0.00 \mathrm{a}$ & $0.00 \mathrm{a}$ & $0.00 \mathrm{a}$ & $0.00 \mathrm{a}$ & $0.00 \mathrm{a}$ & $1.56 \mathrm{a}$ & $0.00 \mathrm{a}$ & $0.00 \mathrm{a}$ \\
\hline Ethanal & 1.70 & $1.54 \mathrm{a}$ & $1.07 \mathrm{a}$ & $2.83 \mathrm{a}$ & $1.27 \mathrm{a}$ & $3.99 \mathrm{a}$ & $2.27 \mathrm{a}$ & $2.84 \mathrm{a}$ & $0.00 \mathrm{a}$ & $2.07 \mathrm{a}$ \\
\hline Methyl acetate & 2.20 & $4.28 \mathrm{a}$ & $4.56 \mathrm{a}$ & $4.83 \mathrm{a}$ & $0.89 a$ & $5.67 b$ & $2.51 \mathrm{a}$ & 3.37 a & $7.09 \mathrm{a}$ & $2.80 \mathrm{a}$ \\
\hline Ethyl acetate & 2.73 & $0.00 \mathrm{a}$ & $69.00 \mathrm{a}$ & $41.62 \mathrm{a}$ & $0.00 \mathrm{a}$ & $38.80 \mathrm{~b}$ & $12.80 \mathrm{a}$ & $0.00 \mathrm{a}$ & $54.73 \mathrm{~b}$ & $24.88 a b$ \\
\hline Ethyl propanoate & 3.53 & $0.00 \mathrm{a}$ & $0.00 \mathrm{a}$ & $1.56 \mathrm{a}$ & $0.00 \mathrm{a}$ & $1.87 \mathrm{a}$ & $1.36 \mathrm{a}$ & $0.00 \mathrm{a}$ & $0.00 \mathrm{a}$ & $0.00 \mathrm{a}$ \\
\hline Propyl acetate & 3.80 & $0.00 \mathrm{a}$ & 23.47 b & $4.34 \mathrm{a}$ & $0.00 \mathrm{a}$ & $15.66 \mathrm{~b}$ & $2.04 \mathrm{a}$ & $0.00 \mathrm{a}$ & $21.46 \mathrm{~b}$ & $3.29 \mathrm{a}$ \\
\hline Ethyl butanoate & 4.97 & $0.00 \mathrm{a}$ & 11.38 a & $10.83 \mathrm{a}$ & $0.00 \mathrm{a}$ & $8.70 \mathrm{~b}$ & $4.42 \mathrm{ab}$ & $0.00 \mathrm{a}$ & $11.59 b$ & $7.51 \mathrm{ab}$ \\
\hline Butyl acetate & 5.70 & 9.62 a & 45.66 b & $58.85 b$ & $5.34 \mathrm{a}$ & $57.31 b$ & $57.21 \mathrm{~b}$ & $10.50 \mathrm{a}$ & $70.31 b$ & $48.59 \mathrm{~b}$ \\
\hline 2-methylbutyl acetate & 7.01 & $0.00 \mathrm{a}$ & $2.92 \mathrm{~b}$ & $1.53 b$ & $0.00 \mathrm{a}$ & $5.94 b$ & $1.43 \mathrm{a}$ & $0.00 \mathrm{a}$ & $7.07 \mathrm{~b}$ & $1.10 \mathrm{a}$ \\
\hline 3-methylbutyl acetate & 7.02 & $0.00 \mathrm{a}$ & $0.00 \mathrm{a}$ & $1.70 \mathrm{a}$ & $0.00 \mathrm{a}$ & $0.70 \mathrm{a}$ & $1.26 \mathrm{a}$ & $0.00 \mathrm{a}$ & $1.10 \mathrm{a}$ & $0.00 \mathrm{a}$ \\
\hline Pentyl acetate & 8.40 & $0.00 \mathrm{a}$ & $1.08 \mathrm{a}$ & $1.18 \mathrm{a}$ & $0.00 \mathrm{a}$ & $1.07 \mathrm{a}$ & $0.80 \mathrm{a}$ & $0.00 \mathrm{a}$ & $0.67 a b$ & $2.06 \mathrm{~b}$ \\
\hline Heptyl acetate & 8.49 & $0.00 \mathrm{a}$ & $0.50 \mathrm{a}$ & $0.00 \mathrm{a}$ & $0.00 \mathrm{a}$ & $0.49 a$ & $1.24 \mathrm{a}$ & $0.00 \mathrm{a}$ & $0.73 a$ & $0.00 \mathrm{a}$ \\
\hline Methyl hexanoate & 8.80 & $2.64 \mathrm{a}$ & $3.92 \mathrm{a}$ & $7.54 \mathrm{~b}$ & $1.93 \mathrm{a}$ & $4.35 b$ & $5.63 \mathrm{~b}$ & $5.12 \mathrm{a}$ & $5.90 \mathrm{a}$ & $4.32 \mathrm{a}$ \\
\hline $\begin{array}{l}\text { Ethyl hexanoate. } \\
\text { ethyl ester }\end{array}$ & 10.30 & $0.00 \mathrm{a}$ & 11.66 a & 20.61 a & $0.22 \mathrm{a}$ & 10.61 a & $6.52 \mathrm{a}$ & $0.00 \mathrm{a}$ & $13.82 \mathrm{a}$ & $17.66 \mathrm{a}$ \\
\hline Hexyl acetate & 11.50 & $0.85 \mathrm{a}$ & $19.20 \mathrm{~b}$ & $17.68 \mathrm{~b}$ & $0.56 \mathrm{a}$ & $18.51 \mathrm{~b}$ & $13.26 \mathrm{~b}$ & $0.60 \mathrm{a}$ & $27.48 b$ & $13.44 \mathrm{a}$ \\
\hline Octanone & 11.80 & $1.83 \mathrm{~b}$ & $0.42 \mathrm{a}$ & $0.61 \mathrm{a}$ & $1.41 \mathrm{a}$ & $0.46 \mathrm{a}$ & $0.59 a$ & $1.90 \mathrm{~b}$ & $0.00 \mathrm{a}$ & $0.56 \mathrm{a}$ \\
\hline Hexanol & 14.20 & $0.39 a$ & $1.32 \mathrm{~b}$ & $1.70 \mathrm{~b}$ & $0.25 \mathrm{a}$ & $1.69 \mathrm{~b}$ & $1.59 \mathrm{~b}$ & $0.20 \mathrm{a}$ & $1.44 \mathrm{a}$ & $1.57 \mathrm{a}$ \\
\hline Hexyl butanoate & 16.07 & $2.03 \mathrm{a}$ & $0.97 \mathrm{a}$ & $4.57 \mathrm{a}$ & $0.80 \mathrm{a}$ & $0.94 \mathrm{a}$ & $2.77 \mathrm{a}$ & $1.72 \mathrm{a}$ & $3.35 \mathrm{a}$ & $2.01 \mathrm{a}$ \\
\hline Octanol & 16.38 & $11.24 \mathrm{a}$ & $11.51 \mathrm{a}$ & $8.39 \mathrm{a}$ & $9.73 \mathrm{a}$ & $11.28 b$ & $8.26 \mathrm{a}$ & $12.93 \mathrm{~b}$ & $12.35 \mathrm{ab}$ & $8.50 \mathrm{a}$ \\
\hline Ethyl octanoate & 16.66 & $0.00 \mathrm{a}$ & $1.29 \mathrm{~b}$ & $0.67 \mathrm{ab}$ & $0.00 \mathrm{a}$ & $1.21 \mathrm{~b}$ & $0.52 \mathrm{ab}$ & $0.00 \mathrm{a}$ & $1.92 \mathrm{~b}$ & $0.95 a b$ \\
\hline Ethyl hexanoate & 18.40 & $1.33 \mathrm{~b}$ & $1.09 \mathrm{ab}$ & $0.70 \mathrm{a}$ & $1.08 \mathrm{a}$ & $0.96 \mathrm{a}$ & $0.90 \mathrm{a}$ & $1.33 b$ & $1.21 \mathrm{~b}$ & $0.67 \mathrm{a}$ \\
\hline Ethyl decanoate & 22.75 & $0.00 \mathrm{a}$ & $1.40 \mathrm{~b}$ & $0.65 a b$ & $0.00 \mathrm{a}$ & $0.94 \mathrm{~b}$ & $0.36 \mathrm{a}$ & $0.11 \mathrm{a}$ & $2.30 \mathrm{a}$ & $0.27 \mathrm{a}$ \\
\hline \multirow[t]{2}{*}{$\alpha$ farnese } & 25.7 & $0.56 \mathrm{a}$ & $25.07 a b$ & 34.92 b & $0.16 \mathrm{a}$ & $28.81 \mathrm{~b}$ & $23.90 \mathrm{~b}$ & $1.21 \mathrm{a}$ & 52.08 b & $11.15 \mathrm{a}$ \\
\hline & & $38.14 \mathrm{a}$ & $237.49 \mathrm{~b}$ & $227.31 \mathrm{~b}$ & $23.65 \mathrm{a}$ & $219.96 \mathrm{c}$ & $151.67 \mathrm{~b}$ & 43.38 a & $296.60 \mathrm{~b}$ & $153.41 \mathrm{a}$ \\
\hline
\end{tabular}

Means with different letters in the same row indicate significant statistical differences in and between sampling points according to the Tukey test $(P \leq 0.05$; $n=4)$.

between harvest and 24 DAH for: butyl acetate; pentyl acetate; octanone; octanol; ethyl hexanoate; and between 12 and 24 DAH for: propyl acetate; 2-methylbutyl acetate; hexyl acetate; ethyl hexanoate and $\alpha$-farnese.

Once the volatile compounds were identified and quantified, they were separated into 5 groups or chemical families: 1) aldehydes (pentanal and ethanal), 2) esters (methyl acetate; ethyl acetate: ethyl propanoate, propyl acetate, ethyl butanoate, butyl acetate, 2-methylbutyl acetate, 3-methylbutyl acetate, pentyl acetate, heptyl acetate, methyl hexanoate, ethyl hexanoate, hexyl acetate, hexyl butanoate, ethyl octanoate and ethyl decanoate), 3) acetones (octanone). 4) alcohols (hexanol and octanol) and 5) terpenes ( $\alpha$ farnese).

In the treatments, it was found that the compounds with the highest proportion that contributed to aroma included esters, mainly methyl, ethyl and butyl; alcohols such as octanol; and terpenes such as $\alpha$ farnese and ethanal, similar to that found by Griñan et al. (2019) in quince (Cydonia oblonga Mill.) (Tab. 5). These compounds have also been identified in fresh fruits, juices and pear liqueurs by Zlatic et al. (2016) in Bartlett, Sevilla et al. (2011) in pear juice Conference, Zhou et al. (2015) in 'Nanguoli', Li et al. (2014) in 'Pingxiangli' and 'Ruanerli', Li et al. (2012) in 'Ruanerli', 'Kurle Xiangli', 'Bartlett', 'Youhongli', 
Table 5. Content and percentages chemical groups of the volatiles $\left(\mathrm{mg} \mathrm{kg}^{-1} \mathrm{~L}^{-1}\right)$ for the treatments $100 \% E T c, 74 \% E T c$ and $48 \%$ ETc of the pear cultivar Triumph of Vienna.

\begin{tabular}{|c|c|c|c|c|c|c|}
\hline \multirow{2}{*}{ Volatile } & \multicolumn{6}{|c|}{ May 9 (harvest) } \\
\hline & $100 \% \mathrm{ETc}$ & Error & 74\%ETC & Error & $48 \% \mathrm{ETc}$ & Error \\
\hline Aldehydes & 3.38 & 1.96 & 1.27 & 1.27 & 4.40 & 1.61 \\
\hline$\%$ aldehydes & $8.00 \mathrm{aAB}$ & 5.66 & $4.10 \mathrm{aAB}$ & 4.10 & $11.97 \mathrm{aB}$ & 5.33 \\
\hline Esters & 19.42 & 11.50 & 9.75 & 3.27 & 21.31 & 5.35 \\
\hline$\%$ esters & $41.05 \mathrm{aA}$ & 10.55 & $36.08 \mathrm{aA}$ & 12.50 & $46.60 \mathrm{aAB}$ & 6.26 \\
\hline Acetones & 1.83 & 0.06 & 1.41 & 0.14 & 1.90 & 0.33 \\
\hline$\%$ acetones & $6.22 \mathrm{aB}$ & 1.48 & $6.79 \mathrm{aB}$ & 1.77 & $4,58 \mathrm{aB}$ & 0.79 \\
\hline Alcohols & 12.95 & 1.80 & 11.05 & 0.58 & 14.56 & 1.61 \\
\hline$\%$ alcohols & $44.00 \mathrm{aB}$ & 12.35 & $52.45 \mathrm{aB}$ & 11.87 & $34.68 \mathrm{aB}$ & 3.69 \\
\hline Terpenes & 0.56 & 0.56 & 0.16 & 0.16 & 1.21 & 1.10 \\
\hline$\%$ terpenes & $0.73 \mathrm{aA}$ & 0.73 & $0.57 \mathrm{aA}$ & 0.57 & $2.17 \mathrm{aAB}$ & 1.89 \\
\hline Total & $38.14 \mathrm{a}$ & 15.89 & $23.65 \mathrm{a}$ & 5.41 & $43.38 \mathrm{a}$ & 10.00 \\
\hline$\%$ total & 100 & & 100 & & 100 & \\
\hline \multicolumn{7}{|c|}{ May 21 (12 DAH) } \\
\hline Aldehydes & 1.07 & 1.07 & 3.99 & 1.36 & 0.00 & 0.00 \\
\hline$\%$ aldehydes & $0.60 \mathrm{abA}$ & 0.60 & $1.88 \mathrm{bA}$ & 0.64 & $0.00 \mathrm{aA}$ & 0.00 \\
\hline Esters & 195.60 & 27.29 & 171.84 & 22.57 & 227.21 & 37.18 \\
\hline$\%$ esters & $81.34 \mathrm{aC}$ & 3.72 & $77.45 \mathrm{aC}$ & 4.35 & $76.97 \mathrm{aC}$ & 2.29 \\
\hline Acetones & 0.42 & 0.42 & 0.46 & 0.46 & 0.00 & 0.00 \\
\hline$\%$ acetones & $0.24 \mathrm{aA}$ & 0.24 & $0.23 \mathrm{aA}$ & 0.23 & $0.00 \mathrm{aA}$ & 0.00 \\
\hline Alcohols & 15.33 & 0.88 & 14.87 & 0.97 & 17.30 & 1.97 \\
\hline$\%$ alcohols & $6.72 \mathrm{aA}$ & 0.99 & $6.86 \mathrm{aA}$ & 0.50 & $6.08 \mathrm{aA}$ & 0.55 \\
\hline Terpenes & 25.07 & 4.77 & 28.81 & 5.90 & 52.08 & 13.18 \\
\hline$\%$ terpenes & $11.11 \mathrm{aABC}$ & 2.38 & $13.57 \mathrm{aABC}$ & 3.38 & $16.95 \mathrm{aBC}$ & 2.55 \\
\hline Total & $237.49 \mathrm{a}$ & 34.43 & $219.96 \mathrm{a}$ & 31.26 & $296.59 \mathrm{a}$ & 52.34 \\
\hline$\%$ total & 100 & & 100 & & 100 & \\
\hline \multicolumn{7}{|c|}{ June 2 (24 DAH) } \\
\hline Aldehydes & 2.83 & 1.22 & 2.27 & 0.92 & 2.07 & 1.20 \\
\hline$\%$ aldehydes & $1.31 \mathrm{aA}$ & 0.49 & $1.61 \mathrm{aA}$ & 0.54 & $1.84 \mathrm{aA}$ & 1.34 \\
\hline Esters & 177.51 & 46.40 & 113.79 & 30.50 & 128.61 & 44.24 \\
\hline$\%$ esters & $74.36 \mathrm{aC}$ & 9.53 & $69.61 \mathrm{aBC}$ & 11.46 & $79.99 \mathrm{aC}$ & 6.15 \\
\hline Acetones & 0.61 & 0.36 & 0.59 & 0.34 & 0.56 & 0.34 \\
\hline$\%$ acetones & $0.37 \mathrm{aA}$ & 0.23 & $0.59 \mathrm{aA}$ & 0.39 & $0.77 \mathrm{aA}$ & 0.46 \\
\hline Alcohols & 11.44 & 0.22 & 11.12 & 0.72 & 11.01 & 0.71 \\
\hline$\%$ alcohols & $5.46 \mathrm{aA}$ & 0.93 & $8.37 \mathrm{aA}$ & 2.03 & 9.56 aA & 2.72 \\
\hline Terpenes & 34.92 & 14.25 & 23.90 & 9.42 & 11.15 & 4.47 \\
\hline$\%$ terpenes & $18.50 \mathrm{aC}$ & 8.37 & $19.82 \mathrm{aC}$ & 9.32 & $7.84 \mathrm{aABC}$ & 3.95 \\
\hline Total & $227.31 \mathrm{a}$ & 35.43 & $151.68 \mathrm{a}$ & 26.18 & $153.40 \mathrm{a}$ & 46.79 \\
\hline$\%$ total & 100 & & 100 & & 100 & \\
\hline
\end{tabular}

Means with different lowercase letters in the row of sampling point and different capital letters between the sampling point of the treatments indicate different statistics according to the Tukey test $(P \leq 0.05)(n=4)$. 
'Daxiangshui', 'Xiaoxiangshui', 'Nanguoli', 'Pingxiangli', 'Dongguoli', 'Hongxiangsu' and 'Zaobaimi', Cano-Lamadrid et al. (2018a) in Pomegranates, CanoLamadrid et al. (2018b) in citrus and Andreu-Coll et al. (2020) in Prickly pear fruit.

With the imposition of the deficit, the water potential at dawn ( $\Psi \mathrm{a})$ and the midday stem water potential $(\Psi \mathrm{t})$, as expected, tended to decrease: the smallest regime (48\%ETc) had $\Psi$ a values of $-0.29 \mathrm{MPa}$ and $\Psi \mathrm{t}$ values of -0.80 and $-0.99 \mathrm{MPa}$ in 2014 and 2015 , respectively. $74 \% \mathrm{ETc}$ had similar values of $\Psi \mathrm{Ya}(-0.26$ $\mathrm{MPa})$ and $\Psi \mathrm{t}(-0.78$ and $-0.96 \mathrm{MPa})$ in 2014 and 2015, respectively, while, in the control, the $\Psi$ a was -0.30 $\mathrm{MPa}$, and the $\Psi \mathrm{t}$ was -0.70 and $-1.03 \mathrm{MPa}$ in 2014 and 2015 , respectively.

The different water regimes resulted in changes in the concentrations of the principle compounds and chemical families of volatiles, with no differences at harvest, $12 \mathrm{DAH}$ or $24 \mathrm{DAH}$, except the percentage of aldehydes at $12 \mathrm{DAH}$ (Tab. 5).

The low total concentrations of the main chemical families form the aroma of fresh and processed fruits (Sevilla et al., 2011), which, in the cultivar Triumph of Vienna, is characteristically sweet and fruity with an herbaceous smell resulting from the dominance of esters, which had the greatest contribution to aroma for the volatile compounds in all treatments, which increased steadily during the climacteric phase and increased in concentration with maturity, while the alcohols decreased as did the aldehydes although the latter did so significantly in 48\%ETc (Wang et al., 2011; Altisent et al., 2011).

As the fruit began to mature, the content of esters increased drastically in all the treatments, which resulted in an increase in aroma, similar to that found in pears by Li et al. (2013). The percentages of esters reached their maximum in deficit treatment $48 \%$ ETc during maturation, coinciding with the increase in the production of ethylene. At harvest, the predominant compounds were esters, alcohols and aldehydes, which decreased after $12 \mathrm{DAH}$, with an increase in the percentage of terpenes (Li et al., 2014; Sevilla et al., 2011). At $24 \mathrm{DAH}$, the predominants were esters, terpenes and alcohols (Tab. 5). $\alpha$-farnese was detected in P. communis by Yanine et al. (2013), similar to that found at $24 \mathrm{DAH}$ for terpenes. The esters were the dominant volatile in deficit treatment $48 \% \mathrm{ET}$ : at harvest (46.0\%); $12 \mathrm{DAH}(76.97 \%)$ and $24 \mathrm{DAH}$ (79.99\%), while, at harvest, $100 \%$ ETc and $74 \% E T c$ presented a higher percentage of alcohols (Tab. 5).

\section{CONCLUSION}

The production of volatile compounds in all treatments increased during maturation and slowed down at $24 \mathrm{DAH}$, possibly resulting from a restricted supply of substrates for esterification. This behavior is typical of climacteric fruits, which produce a greater quantity of compounds with characteristic intense aromas during ripening, coinciding with a high production of ethylene and high respiration, which produce several physiological changes because of a higher concentration of sugars but a lack of precursors at the end of the maturation process.

In the pear cultivar Triumph of Vienna, the volatile compounds with the highest proportion that contributed to aroma were esters, mainly methyl, ethyl and butyl; alcohols such as octanol; terpenes such as $\alpha$-farnese and ethanal.

The different water regimes resulted in changes in the concentrations of the principle volatile compounds without differences between harvest, $12 \mathrm{DAH}$ and 24 $\mathrm{DAH}$. There were also no differences in the percentages of volatile content between the chemical groups at harvest, $12 \mathrm{DAH}$ and $24 \mathrm{DAH}$, except at $12 \mathrm{DAH}$ between treatments 74\%ETC and 48\%ETC for the percentage of aldehydes.

Under limited water conditions, watering with regulated deficit doses can provide production that is similar to that of a well-watered crop, provided that it is carried out in the phenological state of low sensitivity and the tolerance limits of stress are not exceeded.

Conflict of interests: the manuscript was prepared and reviewed with the participation of all the authors, who declare that there exists no conflict of interest that puts at risk the validity of the presented results.

\section{BIBLIOGRAPHIC REFERENCES}

Agronet, Red de Información y Comunicación del Sector Agropecuario Colombiano. 2019. Área, producción y rendimiento por cultivo. In: Ministerio de Agricultura y Desarrollo Rural, https://www.agronet.gov.co/estadistica/Paginas/home.aspx?.cod=1; consulted: December, 2019 .

Allen, R.G., L.S. Pereira., D. Raes, and M. Smith. 1998. Crop evapotranspiration, guidelines for computing crop water requirements. Irrigation and Drainage Paper 56. FAO, Roma. 
Altisent, R., J. Graell., I. Lara, L. López, and G. Echeverria. 2011. Comparison of the volatile profile and sensory analysis of 'Golden Reindeers' apples after the application of a cold air period after ultralow oxygen (ULO) storage. J. Agric. Food Chem. 59, 6193-6201. Doi: 10.1021/jf2005029

Andreu-Coll, L., L. Noguera-Artiaga, A. Carbonell-Barrachina, P. Legua, and F. Hernández. 2020. Volatile composition of prickly pear fruit pulp from six Spanish cultivars. J. Food Sci. 85(2), 358-363. Doi: 10.1111/1750-3841.15001

Bangerth, F.K., J. Song, and J. Streif. 2012. Physiological impacts of fruit ripening and storage conditions on aroma volatile formation in apple and strawberry fruit. HortScience 47, 4-10. Doi: 10.21273/HORTSCI.47.1.4

Bhavadharani, R.K., V, Nagarajan, and R. Chandiramouli. 2019. Silicene nanosheet to discriminate the quality of pear fruit based on volatiles adsorption ---a DFT application. Condens. Matter Phys. 22 (3), 33001. Doi: 10.5488/CMP.22.33001

Cano-Lamadrid, M., A., Galindo, J. Collado-Gonzáles, P. Rodriguez, Z.C. Cruz, P. Legua, F. Burló, D. Morales, A. Carbonell-Barrachina, and F. Hernández. 2018. Influence of deficit irrigation and crop load on the yield and fruit quality in Wonderful and Mollar de Elche pomegranates. J. Sci. Food Agr. 98, 3098-3108. Doi: $10.1002 /$ jsfa. 8810

Cano-Lamadrid, M., L, Lipan., F, Hernández., J. J, Martínez., P, Legua., A. Carbonell-Barrachina, and P, Melgarejo. 2018. Quality parameters, volatile composition, and sensory profiles of highly endangered spanish citrus fruits. J. Food Qual. 2018, 3475461. Doi: $10.1155 / 2018 / 3475461$

Cleves-Leguizamo, J.A., J. Toro-Calderón, and L. Martínez-Bernal. 2016. Los balances hídricos agrícolas en modelos de simulación agroclimáticos. Una revisión analítica. Rev. Colomb. Cienc. Hortic. 10(1), 149-163. Doi: 10.17584/rcch.2016v10i1.4460

Galindo, A., A. Calín-Sánchez., I. Griñan., P. Rodríguez., Z.N. Cruz., I. F. Girón., M. Corell., R. Martínez-Font., A. Moriana., A. Carbonell-Barrachina., A. Torrecillas, and F. Hernández. 2017. Water stress at the end of the pomegranate fruit ripening stage produces earlier harvest and improves fruit quality. Sci. Hortic. 226, 6874. Doi: 10.1016/j.scienta.2017.08.029

Griñan, I., A. Galindo., P. Rodríguez., D. Morales., M. Corell., J. Centeno., J. Collado-Gonzales., A. Torrecillas., A. Carbonell-Barrachina, and F. Hernandez. 2019. Volatile composition and sensory and quality attributes of quince (Cydonia oblonga Mill.) fruits as affected by water stress. Sci. Hort. 244, 68-74 Doi: 10.1016/j. scienta.2018.09.013

IGAC, Instituto Geográfico Agustín Codazzi. 2010. Clasificación de suelos en el departamento de Cundinamarca.
Universidad Tecnológico y Pedagógica de Colombia, Departamento Nacional de Estadística; IGAC subdirección de Agrología 169, 325-327.

Li, G., H. Jia., R. Wu., S. Hussain, and Y. Teng. 2012. Characterization of aromatic volatile constituents in 11 Asian pear cultivars belonging to different species. Afr. J. Agric. 7, 4761-4770. Doi: 10.5897/AJAR12.563

Li, G., H. Jia., R. Wu, and Y. Teng. 2013. Changes in volatile organic compound composition during the ripening of 'Nanguoli' pears (Pyrus ussuriensis M.) harvested at different growing locations. J. Hortic. Sci. Biotechnol. 88 (5), 563-570. Doi: 10.1080/14620316.2013.11513007

Li, G.P., J.H. Jia., Q. Li., M.J. Wang, and Y.W. Zhang. 2014. Emission of volatile esters and transcription of ethylene- and aroma-related genes during ripening of 'Pingxiangli' pear fruit (Pyrus ussuriensis M.). Sci. Hortic. 170, 17-23. Doi: 10.1016/j.scienta.2014.03.004

Miranda, D., G. Fischer, and C. Carranza. 2013. Los frutales caducifolios en Colombia. Sociedad Colombiana de Ciencias Hortícolas, Bogota.

Morandi, B., P. Losciale., L. Manfrini., M. Zibordi., S. Anconelli., F. Galli., E. Pierpaoli, and L. Corelli. 2014. Increasing water stress negatively effects pear fruit growth by reducing first its xylem and then its phloem inflow. J. Plant Physiol. 171(16), 1500-1509. Doi: 10.1016/j. jplph.2014.07.005

SAFC. 2011. SAFC Flavors and fragrances catalog. Sigma-Aldrich, Madrid.

SAS Institute. 2010. Statistical analysis system version 8 for Windows Inc. Cary, NC.

Sevilla, A., A. Carbonell., J. López, and F. García. 2011. Comparative effect of the addition of $\alpha-, \beta$-, or $\gamma$-cyclodextrin on main sensory and physico-chemical parameters. J. Food Sci. 76(5), 347-353. Doi: 10.1111/j.1750-3841.2011.02190.x

Stashenko, E, and J. Martínez. 2011. Preparación de la muestra: un paso crucial para el análisis y GC-MS. Scientia Chromatographica 3(1), 25-49. Doi: 10.4322/ sc. 2011.003

UNESCO, United Nations Educational, Scientific and Cultural Oorganization. 2015. Science Report: towards 2030. Executive Summary. 2015. Water for a sustainable world the United Nations world water development report.

Verzera, A., G. Dima., G. Tripodi., M. Ziino., C. Lanza, and A. Mazzaglia. 2011. Fast quantitative determination of aroma volatile constituents in melon fruits by headspace-solid-phase microextraction and gas chromatography-mass spectrometry. Food Analytical Methods. 4, 141-149. Doi: 10.1007/s12161-010-9159-z

Wang, M. Y., E. Macrae., M. Wohlers, and K. Marsh. 2011. Changes in volatile production and sensory quality of kiwifruit during fruit maturation in Actinidia 
deliciosa 'Hayward' and A. chinensis 'Hor 100\%ETc6A'. Postharvest Biol. Technol. 59, 16-24. Doi: 10.1016/j. postharvbio.2010.08.010

Yanine, A.G., L. García., C.J.R. Pérez., V.C. Lopez., I. Orriols, and F. Lopez. 2013. Aromatically enhanced pear distillates from Blanquilla and Conference varieties using a packed column. J. Agric. Food Chem. 61(20), 4934942. Doi: 10.1021/jf304619e

Zhou, X., L. Dong., Q. Zhou., J. Wang., N. Chang., Z. Liu, and S. Ji. 2015. Effects of intermittent warming on aroma-related esters of 1-methyllcyclopropenetreated 'Nanguo' pears during ripening at room temperature. Sci. Hortic. 185, 82-89. Doi: 10.1016/j. scienta.2015.01.021

Zlatic, E., V. Zadnikb., J. Fellmanc., L. Demsara., J. Hribara., Z. Cejíc, and R. Vidrih. 2016. Comparative analysis of aroma compounds in 'Bartlett' pear in relation to harvest date, storage conditions, and shelf life. Postharvest Biol. Technol. 117, 71-80. Doi: 10.1016/j. postharvbio.2016.02.004 